\title{
LE PATOLOGIE DI GIUSTINIANO E LE PROFILASSI MEDICHE IN VOGA NELLA PROTO BISANZIO. GLI ADYNATA COME ECCEZIONALE CURA
}

\author{
Antonio Pio Di Cosmo \\ ISACCL, Bucarest \\ Email: apiocosmo@outlook.it \\ ORCID iD: https://orcid.org/0000-0002-2314-4520
}

Recibido: 7 diciembre 2019; Aceptado: 2 enero 2021

Cómo citar este artículo/Citation: Di Cosmo, Antonio Pio (2021) "Le patologie di Giustiniano e le profilassi mediche in voga nella Proto Bisanzio. Gli Adynata come eccezionale cura”, Asclepio, 73 (1): p336. https://doi.org/10.3989/asclepio.2021/02

RIASSUNTO: Questa ricerca confronta i dati raccolti dai testi riguardanti l'imperatore Giustiniano con le informazioni concernenti la casistica delle malattie sessuali nel contesto della Proto Bisanzio. Un testo agiografico, la Vita di San Sansone, racconta: una grave malattia colpisce Giustiniano agli organi genitali e i medici non sono in grado di curarla. La patologia viene risolta da un miracolo di San Sansone. Questo adynaton pone l'imperatore in uno 'spazio di copertura'. Dopo il $23^{\circ}$ o $24^{\circ}$ anno del suo regno, Giustiniano soffre disturbi ad una gamba, accompagnati da dolore durante la minzione. Körbler ipotizza che questi sintomi siano dovuti alla sifilide. Il disturbo alla gamba viene curato dall'intercessione dei santi Cosma e Damiano. La malattia dell'imperatore si rivela dunque come luogo della letteratura agiografica. L'adynaton poi conferma l'istituzione imperiale ed afferma l'adeguatezza del sovrano.

PAROLE CHIAVE: Giustiniano; sifilide; malattie sessuali; adynata; San Cosma e Damiano.

\section{JUSTINIAN'S PATHOLOGIES AND MEDICAL PROPHYLAXIS IN THE EARLY BYZANTIUM. ADYNATA AS AN EXCEPTIONAL CARE}

ABSTRACT: This research compares the collected data from texts regarding the life of Emperor Justinian with information on cases of sexual diseases in the Early Byzantium contest. A hagiographical text, The Life of Saint Samson, recounted: a serious illness affected Justinian to the genital organs and doctors didn't have a care. The disease was resolved by a miracle of San Samson. This adynaton placed the Emperor in a coverage space. After, in the 23 rd or 24th year of his reign, Justinian suffered a leg pain, accompanied by pain during the urination. Körbler hypothesized that these symptoms were due to syphilis. The disturbance at the leg was cured by the intercession of Saints Cosma and Damian. Rather the emperor's illness was detected as a literary locus of hagiography. The adynaton confirms the foundation of the imperial institution and affirms the adequacy of the sovereign.

KEYWORDS: Justinian; Syphilis; Sexual Disease; Adynata; Saint Cosma and Damian. 


\section{INTRODUZIONE}

La presente indagine pone in essere la collazione dei dati estrapolati dalle diverse fonti testuali che fanno menzione dell'imperatore Giustiniano (482-565) e alludono ad alcune patologie che hanno afflitto la sua esistenza. Fra esse particolare importanza riveste un testo agiografico: la Vita di San Sansone (? -530) fondatore di un ospedale in Costantinopoli, che sovente interagisce con questo sovrano ed esprime il proprio potere taumaturgico per rimediare ad una patologia a cui i medici di palazzo non riescono ad opporre alcuna cura. Deve poi tenersi in particolare conto quella sorta di biografia 'non ufficiale', nonché malevola interpretazione del vissuto di Giustiniano e della moglie Teodora, che è la Historia Arcana di Procopio di Cesarea (490565). Questo perché i prefati testi offrono una serie di informazioni fondamentali per meglio delineare un ritratto a tutto tondo di questa coppia, che prescinde dalla lusinghiera letteratura di corte (Kaldellis 2004). Eppure queste fonti 'non ufficiali' non si limitano solo a quello. Forniscono una serie di dati che gli storici e gli studiosi di medicina in particolare possono adoperare per valutare lo stato delle conoscenze mediche nella Proto Bisanzio ed, altresì, per constatare l'esistenza dei relativi rimedi a diversi tipi di patologia.

Alla luce di quanto evinto dalle fonti collazionate, si procede ad un'integrazione dei dati concernenti sia la più generica biografia di Giustiniano, sia le probabili patologie da lui sofferte, sia le profilassi mediche eseguite per curarne i sintomi, comparandole con le più generali informazioni evincibili dai trattati di medicina del periodo, che analizzano casi medici assimilabili e propongono cure.

Deve precisarsi che questi testi 'non ufficiali' introducono un ulteriore tema per il pubblico dei loro lettori: I'adynaton. Ben si comprende come l'intervento divino si dimostra indispensabile per la monarchia cristiana, quale motivo legittimante e posto a garanzia della bontà dell'azione di governo esercitata. Questo, difatti, spiega l'adeguatezza del sovrano a detenere I'Impero. II miracolo, operato nella vita dell'augusto, offre una prova incontestabile del consenso divino che supporta l'imperatore romano e si addice al contesto in cui sviluppa il racconto agiografico. Diventa così indispensabile per l'apologia della monarchia e fornisce pregevolezza alla condotta di un augusto soggetto ad aspra critica come Giustiniano. Tuttavia, l'adynaton è un luogo tradizionale in qualsiasi biografia imperiale. E se ciò fa sorgere dubbi sulla credibilità dell'episodio narrato in sé, non compromette però l'attendibilità dei dati concernenti la prassi medica seguita. Poiché pare difficile sostenere che i diversi autori si siano inventati di sana pianta cure mediche, che appaiono ictu oculi troppo eccentriche, almeno rispetto a quelle a cui è abituato il pubblico. Nondimeno, la credibilità dell'accadimento meraviglioso, almeno rispetto alle conoscenze mediche del periodo, viene avvalorata da un'opera di aspra critica come la Historia Arcana. II suo inserimento dimostra che un tale fatto deve essere universalmente noto e non può perciò essere né contestato, né sottaciuto.

Si comprende bene come la malattia sofferta dall'imperatore possa divenire un ulteriore luogo dell'agiografia, che permette ai santi di dimostrare il proprio potere taumaturgico e di operare cose meravigliose, capaci di avvallare la loro fama. II meraviglioso svolge un'altra funzione: ottimizza i processi di comunicazione che diffondono i valori della Chiesa. Eppure, non solo la Chiesa trova giovamento dagli accidenti della vita di questo imperatore. Come si è già efficacemente sottolineato, è lo stesso Impero che trae profitto da questi racconti capaci di unire in un connubio la malattia del sovrano, anche se imbarazzante, all'intervento divino. L'azione della divinità permette all'istituzione di recuperare la sua autorevolezza dal punto di vista ideologico, che quella specifica malattia può aver messo in discussione.

Nondimeno, la presunzione della contrazione di una malattia sessuale costituisce un argomento sensibile, che esige sempre l'uso di precauzioni. Cosa che spiega la sua ragionevole espunzione dalle biografie imperiali 'ufficiali'. E se la presunta malattia sessuale sofferta dall'imperatore Giustiniano diventa un motivo edificante nell'agiografia, non appare affatto spendibile sul piano politico e può persino offrire pericolosi spunti retorici alla kaiserkritik. La sua sommessa evocazione in opere sostanzialmente ostili come la Historia Arcana costituisce, dunque, un'occasione di espressione di dissenso da parte dell'aristocrazia, che commissiona simili produzioni letterarie. Dissenso a cui il meraviglioso, che non può essere ignorato, pone rimedio.

\section{GONORREA E SIFILIDE, LA PROTO BISANZIO E LO STATO DELLE CONOSCENZE MEDICHE A RIGUARDO}

La serie di trattati medici in circolazione nella cronologia di riferimento permette di affermare senza dubbio che lo stato delle conoscenze concernenti le malattie sessuali, dimostrato dai medici romano orientali, è molto elevato e la speculazione teorica sulle problematiche persino raffinata. Eppure ciò stride con l'effettiva capacità dei medici di porre rimedio alle patologie, poiché basta confrontare le elaborazioni della 
dottrina medica con i mezzi tecnici che gli stessi operatori sanitari possiedono. Questi medici, difatti, soffrono l'assenza di strumenti endoscopici, nonché dell'impossibilità di analizzare la natura delle secrezioni degli organi genitali. Strumenti indispensabili per capire il fattore eziologico della malattia. Deve precisarsi che al tempo molti sintomi delle infermità dell'apparato genitale-urinario sono ben noti ai medici, come ex multis i sintomi dell'uretrite. Eppure, allorché si discerne delle patologie dell'apparato genitale ed urinario occorre muoversi con cautela: una serie di differenti malattie può presentare sintomi assimilabili. Ad esempio, l'uretrite è prodotta dal gonococco, ma anche da altri germi. Di conseguenza la conoscenza dei sintomi è indipendente dal suo preciso agente eziologico.

Nondimeno, si osserva che i sintomi di un'altra malattia: la gonorrea sono menzionati già nella Bibbia e sono stati descritti pure da Celso nel I secolo d.C., nonché da Galeno nel II secolo d.C.. ${ }^{1}$

Nondimeno, questi sintomi sono anche esposti nei trattati di Ippocrate nel IV secolo a.C., il quale ha una conoscenza approfondita di questo genere di malattie; costui giunge a descrivere sia la leucorrea parassitaria, sia la leucorrea micotica, sia la leucorrea batterica.

Occorre altresì fare lumi sullo stato delle conoscenze mediche della suddetta cronologia concernenti la sifilide, specie se si considera che in letteratura scientifica circolano molte ipotesi sull'origine di questa malattia in Europa. Le tesi fin ad ora proposte dividono gli storici della medicina e sono oggetto di attuale dibattito. La dottrina, almeno in epoca precoce, ha ritenuto che la sifilide sia giunta in Europa non prima dell'ultimo decennio del XV secolo, portata probabilmente dai marinai di Cristoforo Colombo o comunque da soggetti di ritorno dalle Americhe. Tuttavia, alcuni specialisti hanno proposto una serie di argomentazioni capaci di dimostrare che questa patologia si sia diffusa in epoca precoce e non agli inizi della modernità come ritiene la dottrina dominante. Ad opinione di Hudson la malattia fa la sua prima apparizione durante il Paleolitico quale forma endemica, che assume le caratteristiche della sifilide 'venerea' in luoghi ed in momenti diversi, in concomitanza con l'urbanizzazione della vita rurale (Hudson 1965, pp. 885-901). A suo dire le forme 'indigene' e 'veneree' coesistono in Mesopotamia ed Egitto al più tardi a partire dal 1000 a.C.. La sifilide, cosiddetta 'indigena', secondo questa teoria, si diffonde fra il 900 e l'800 a.C. in Grecia per poi estendersi per l'Europa occidentale durante la dominazione romana; questa singolare patologia si perpetuerebbe infine per tutto il Medioevo. L'ipotesi non viene però confermata dai dati osteoarcheologici in nostro possesso. Ad esempio, alcuna traccia di sifilide è stata trovata sui resti mortali riesumati in Grecia e nelle terre confinanti (Vertue 1983, pp. 277-302). Di conseguenza i dati biologici raccolti dimostrano che la sifilide non è presente nella Grecia 'classica'. Un dato inequivocabile, che sconfessa una simile ipotesi. Al contrario la lebbra e la tubercolosi vengono ampiamente attestate dall'analisi delle sepolture della relativa cronologia. Grmek, pertanto, può ritenere che la sifilide sia del tutto assente (Grmek 1989, pp. 133-151). Venuti meno i dati a favore della pregevolezza dell'ipotesi di Hudson, diventa davvero difficile supportare l'idea di una presenza della sifilide nella Proto Bisanzio, ma anche nel Tardo Antico in generale.

\section{TEODORA ED IL PREGIUDIZIO DELL'ARISTOCRAZIA: GLI ANEDDOTI MALIZIOSI A TUTELA DEI VALORI DELLA CLASSE}

La scelta matrimoniale del nōbelissimos Giustiniano, che si orienta insolitamente verso una ballerina, a scapito delle consuete politiche matrimoniali della classe nobiliare, ha suscitato non poca curiosità ed ilarità nell'aristocrazia: il gruppo dei pari. Questa 'stranezza', comprensibilmente, deve lasciare perplesse anche le classi medio-alte del tempo. ${ }^{2}$ Tale decisione non può essere perdonata né dai ben pensanti, né dai più conservatori tra i patrizi, tanto che viene usata come pretesto per un'incisiva kaiserkritik. Questa appare ai propri pari come una violazione dei valori della classe ed una irrispettosa negazione dell'esclusività dello status detenuto rispetto al resto dei consociati. L'aristocrazia, come ben noto, preferisce forme endogene di matrimonio, perché per tradizione le classi altolocate tendono a conservare l'esclusività del proprio lignaggio e non permettono l'ingresso tra loro di soggetti provenienti dalle classi dei sottoposti. Tale politica nella scelta del coniuge viene ritenuta indispensabile per preservare gli attributi ed, infine, l'unitarietà della classe.

Un'ilarità suscitata dal fatto che Giustiniano dispone di abolire persino la legge con cui si proibisce il matrimonio dei membri della classe senatoria con persone di basso livello sociale ed, in particolare, con attrici e prostitute $(C . J . V, 4,23)$. Questi fa venire meno il più grosso ostacolo posto dalla classe aristocratica e volto a salvaguardare l'esclusività degli attributi di status. ${ }^{3}$ Questa violazione dei precetti, che tengono insieme la classe dominante e la rendono quel che è, sottende un cambiamento nella percezione dei valori dell'aristocrazia all'interno della classe stessa.

Non meraviglia che questa scelta matrimoniale abbia suscitato la nascita di aneddoti fantasiosi e male- 
voli, volti a denigrare Giustiniano a mezzo del biasimo verso la sua sposa e le di lei condotte. Questa polemica costituisce una vera e propria reazione posta in essere dall'aristocrazia, seppur sommessa, avverso il tentativo di democraticizzazione degli attributi di classe, preservandone così l'esclusività.

Un'opera denigratoria che viene agevolata da un pregiudizio diffuso: nel mondo antico attori e ballerini sono considerati solitamente persone di dubbia moralità. Una tale concezione sopravvive nel Tardo Antico ed è ereditata dalla Proto Bisanzio. Pertanto nemmeno Teodora (497-548), che ha svolto queste attività in gioventù, può sfuggire a tali critiche seppur è divenuta imperatrice (Ravegnani 2016, pp. 25-33). Se ne deduce che né la porpora, né la corona possono costituire una garanzia assoluta di virtù del detentore, nonostante $\mathrm{i}$ perì basileias hanno già fatto proprio questo locus e cercano di somministrare tale idea ad un pubblico in espansione; quello che ovviamente è capace di leggere tali opere. ${ }^{4}$ Non c'è da stupirsi che i dubbi circa la sua controversa moralità siano caldeggiati da parte di quell'aristocrazia, la quale vede l'entrata tra le proprie file di una persona di umili origini come una minaccia. La più ovvia soluzione è denigrarla evocando situazioni capaci di evidenziare la poca credibilità della sua integrità morale, poiché questa è ritenuta da sempre un attributo proprio delle nobildonne romane; virtù che le distingue dalle popolane.

Una critica in fin dei conti miope e volta soprattutto a censurare il ruolo disinvolto di costei, in quanto è la prima donna che non si fa scrupolo ad intervenire direttamente nel governo dell'Impero. Si comprende allora come Procopio proceda a censurare tale pretesa: "riteneva di dover regolare tutto, nello Stato, di sua iniziativa". La sua condotta dopotutto è stigmatizzata perché viene intesa come una vera e propria violazione delle prerogative dello sposo. Specie allorché questa riceve in assenza del marito e nei propri appartamenti le delegazioni straniere; prassi che non ha precedenti ed apre a tendenziose interpretazioni (Ravegnani 1989, p. 50). Puntualizza Procopio: "Costei non esitava a ricevere gli ambasciatori dei Persiani e degli altri barbari e a far loro doni in denaro come se fosse la padrona dell'Impero dei romani, cosa mai vista sin dall'inizio dei tempi" (Proc. Caes., Hist. Arc. XXX, 21-26).

Tuttavia, non può negarsi che è proprio la sua costante presenza nelle azioni di governo e la sua prontezza a porre fine alla crisi causata dalla 'rivolta di Nika'. Lo stesso Procopio ha cura di riportare l'accorato appello della donna, che la vede esordire contro ogni convenzione sociale del periodo: "Ritengo che nella situazione presente sia irrilevante tener conto della sconvenienza che una donna mostri coraggio fra gli uomini e proponga soluzioni ardimentose a chi ha paura, sia che si pensi così, sia in altro modo...". Questa poi chiude l'intervento con un suggestivo inciso: "Che io non sia mai priva di questa porpora (...). A me infatti piace un antico detto, secondo il quale la veste regale è un bel sudario" (Proc. Caes., De bell. Pers. I, 24).

Una presa di posizione forse irrituale in una riunione del consiglio dei dignitari, eppure efficacissima, che salva col regno le vite dei sovrani. Nonostante la sua risoluzione e la sua indubbia capacità di problem solving (potremmo dire in termini moderni), il dubbio circa le buone intenzioni di Teodora perdura. Si insinua anche quando l'imperatrice si prodiga in opere di misericordia, come la liberazione delle prostitute, sostenuta con apposita novella del 535, volta a proibire il lenonicinio ed a temperare la condizione della donna di medio e basso rango a Bisanzio. Più tardi Malala riferisce: "in quell'anno la pia Teodora aggiunse questa alle sue buone azioni. I così detti lenoni raggiungevano ogni luogo alla ricerca di poveri che avessero figlie, si dice, dando loro garanzie e poche monete d'oro le portavano via (...) -finché l'imperatrice ordina- che questi lenoni fossero arrestati e, messili a confronto con le fanciulle, impose ad ognuno di loro di dichiarare sotto giuramento quanto avevano dato ai loro genitori ed essi risposero di aver dato cinque monete d'oro per ciascuna. E avevano tutti affermato sotto giuramento, la pia imperatrice restituì il denaro e liberò dal giogo di squallida servitù e ordinò che in seguito non vi fossero lenoni..." (Mal., 440-441).

Un'opera buona che non esenta Procopio da un'interpretazione malevola. Ciò a riprova della duttilità del mezzo retorico, allorché si stigmatizza la futilità di una conversione forzosa di quelle donne, che esercitano il mercimonio del loro corpo professionalmente. Costoro, altrettanto forzosamente, vengono relegate presso il cosiddetto Monastero del Pentimento. Procopio afferma:

radunò più di cinquecento meretrici che sulla pubblica piazza si vendevano per tre oboli tanto per sopravvivere e le mandò nel continente di fronte, le rinchiuse nel monastero detto del Pentimento costringendole a mutar vita. Senonché alcune di loro di notte si gettarono dall'alto, liberandosi così da quella conversione forzosa (Proc. Caes., Hist. Arc. XVII, 5-6; XXX, 24).

Una notizia così tendenziosa, come quella delle donne che si sono suicidate gettandosi dalle mura dello stesso convento in cui vengono rinchiuse per sottrarle alla vita di strada, non può essere liquidata come un'informazione di poco valore. Ma è politicamente orientata a denigrare la sovrana. Procopio porta pure 
avanti una critica sociologica, che in fin dei conti offre una visione satirica dei sottoposti a tutto vantaggio della superiorità morale dell'aristocrazia. Mostra poi una certa misoginia di classe, che si indirizza verso tutte le donne delle classi inferiori. Ne oppone così la condotta deprecabile a quella integerrima delle donne aristocratiche. Ciò fa dei nobili natali e del possesso delle risorse la discriminante, che favorisce l'irreprensibilità morale. L'attacco a Teodora si risolve in ultima analisi in un pamphlet della classe dominante.

Ma vi sono ulteriori ragioni. Questa insieme a Giovanni di Cappadocia ha stimolato l'emanazione di una novella sempre del 535 , che abolisce la venialità delle cariche (Lamma 1947, pp. 80-100). Giustiniano puntualizza il loro intervento nel testo della legge: "tutto ciò meditiamo fra di noi e avendo fatto partecipe del proposito la piissima consorte dataci da Dio e la tua sublimità (Giovanni di Cappadocia), da cui abbiamo ricevuto consigli, siamo giunti alla presente divina legge" (Nov. Just. 14a 535).

Una legge che colpisce il monopolio plutocrate e costringe gli esponenti dell'aristocrazia a contrattare, di volta in volta, la propria posizione con la famiglia imperiale per la progressione nel cursus honorum. Motivo più che legittimo per stimolare il dissenso di una classe, che si vede attaccata nelle proprie prerogative, come l'accaparramento dei titoli aulici in ragione della disponibilità delle risorse; privilegio che vede ora messo in discussione.

Non meraviglia nemmeno che tale situazione possa favorire la circolazione di aneddoti imbarazzanti concernenti l'imperatrice, i quali non si limitano alla sua mera attività di ballerina. Sembra che la giovane Teodora oltre a recitare nel teatro dell'ippodromo, abbia lavorato anche in molti bordelli. Durante questa attività in alcune delle città dell'Est, Teodora probabilmente può aver contratto qualche malattia sessuale. Questa tradizione negativa vuole che il padre di Teodora, Acacio, un domatore di bestie selvagge, abbia costretto le sue due figlie, Comitò e Teodora, a prostituirsi. Questa malevola fama che accompagna l'imperatrice favorisce le illazioni dell'aristocrazia. Si arriva a sostenere che Teodora sin da bambina sia stata coinvolta in opere di meretricio e, non essendo ancora matura a tale scopo, abbia intrattenuto rapporti carnali con i clienti assimilabili alla pederastia. Procopio di Cesarea, nella sua opera Historia Arcana, non è certo parco di notizie ed offre dettagli scandalosi sulla vita di Teodora prima del suo matrimonio (Wirth; Haury 1963, pp. 283-284). Innanzitutto Procopio ha a definire Teodora una cortigiana da "truppa", ossia una di quelle che si concedono prevalentemente alle trasgres- sioni biasimate dalla morale comune, anche perché "non sapeva suonare flauto, né arpa e neppure era abile nella danza". Eppure, ella è in grado di offrire la bellezza, bene non meno pregevole. Tuttavia, la giovane donna sembra avere successo nell'arte del mimo, che accompagna all'arte del saper fare l'amore ed alla mancanza di pudore nel rivolgersi agli altri. Procopio stigmatizza gli eccessi della sua condotta: "nessuno la vide mai vergognarsi, al contrario si prestava senza alcuna esitazione a turpi servizi ed era capace di lasciarsi percuotere e schiaffeggiare sulle guance scherzando e ridendoci sopra e, dopo essersi spogliata davanti e dietro, mostrava nude a chiunque quelle parti del corpo che la decenza vuole siano nascoste alla vista degli uomini. Con gli amanti era piena di scherzi e civettuola e, provocandoli con sempre nuove forme di accoppiamento, riusciva a legarsi per sempre l'affetto dei dissoluti (...). Pur usando tre orifizi del suo corpo, se la prendeva con la natura perché non le aveva reso piú larghi i fori dei capezzoli in modo da poter inventare anche là un'altra forma di rapporto (...). -Al contempo, la donna nello svolgere gli spettacoli teatrali non ha a risparmiare affatto l'esibizione del suo corpo, sicché- "spesso anche a teatro, sotto gli occhi di tutto il pubblico, si spogliò e rimase nuda al centro della scena, portando soltanto un perizoma intorno al sesso e all'inguine, non perché si vergognasse di mostrare al popolo anche quelle parti, ma perché qui da noi è vietato a chiunque presentarsi in scena completamente nudo, senza almeno un perizoma intorno all'inguine. In quella condizione si stendeva supina a terra. Allora alcuni servi ai quali era stata affidata l'incombenza le gettavano chicchi d'orzo sul pube, che alcune oche ammaestrate allo scopo beccavano uno a uno per mangiarseli. Quando si alzava, non solo non dava segni di imbarazzo, ma sembrava assai soddisfatta dell'esibizione (...). A lei infine si avvicinavano soltanto i depravati (...). Le persone per bene che la incontravano in piazza si affrettavano per evitare, anche con il semplice contatto con le sue vesti di apparire contaminate da quell'abominio. Era un brutto segno vederla, soprattutto all'inizio della giornata" (Proc. Caes., Hist. Arc. IX, 2- 26).

Sembra poi che verso il 518 Teodora sia diventata I'amante di Ecebolo, un cittadino di Tiro, il quale, divenuto governatore di Pentapoli in Libia, I'ha portata con sé. Terminata la relazione, la donna viene cacciata ed è costretta a pellegrinare in Oriente. In particolare, allorché giunge ad Alessandria può frequentare il patriarca monofisita Timoteo III, che forse la battezza. È pure ad Antiochia, laddove conosce il teologo monofisita Severo.

Procopio sostiene persino la sua partecipazione a festini e, come se non bastasse, ad orge. Durante 
quegli stessi festini -malevolmente afferma il prefato Procopio-, la futura imperatrice ha offerto sollazzo a dieci o più ragazzi ed una volta finito con loro anche ai relativi servi. E così spesso si ritrova in 'stato interessante' e di conseguenza è costretta ad interrompere la gravidanza in vari modi, sovente assumendo gli intrugli che la tradizione e non solo la medicina ufficiale consigliano per il caso. Procopio rivela persino che in un'occasione il tentativo di aborto non ha raggiunto il proprio fine, sicché ha dato alla luce un maschio, che ha chiamato Giovanni; costui sembra poi uscire dalla vita della madre al momento in cui è diventata imperatrice. Stanti tali presupposti non è difficile immaginare che Giustiniano abbia contratto dalla sua futura moglie, che probabilmente ha incontrato in un postribolo, una malattia venerea quale la gonorrea o semplicemente un'infezione batterica alle vie urinarie, che in assenza di antibiotici sono entrambe difficili da curare. È altresì possibile che il futuro imperatore conosca Teodora attraverso una tale Macedonia, una ballerina, la quale l'ha accolta nel suo peregrinare e di cui sappiamo davvero poco. Costei ha intrattenuto una nutrita corrispondenza con Giustiniano ed ha esercitato su di lui una notevole influenza in funzione di delatrice dell'aristocrazia (Proc. Caes., Hist. Arc. IX, 27-28; XII, 28-32).

L'aristocrazia non ha nemmeno perdonato la sua fede: il Nestorianesimo. Un aneddoto relativo si trova nella biografia del santo monaco Saba, che le ha rifiutato preghiere, alla richiesta dell'imperatrice di intercedere per farle ottenere un erede per il trono. II motivo di tale diniego da parte del santo monaco sembra essere il persistere nell'eresia da parte di Teodora. Un'espediente che forma un locus di kaiserkritik davvero pregnante. II rifiuto del miracolo dimostra l'inadeguatezza alla carica della donna di umili origini, che aggiunge ai suoi natali ed alla sua irriverente ingerenza nelle cose riservate agli uomini, anche l'errore in materia di fede.

Questo monaco si reca in visita alla corte imperiale nel 531, su delega di Elia patriarca di Gerusalemme, e chiede sostegno economico per le chiese locali dopo la rivolta dei samaritani. Chiamato da Teodora a colloquio, Saba le rifiuta il miracolo e liquida il suo mancato intervento, dicendo all'imperatrice: "Dio, sovrano e signore di tutte le cose, veglierà sul vostro impero" (Vit. S. Sab. 71).

All'imperatrice non viene perdonato nemmeno il suo gusto per la spettacolarizzazione del potere assoluto che ella detiene. Un comportamento che negli effetti lede le prerogative dell'aristocrazia con atteggiamenti non sempre rispettosi del rango e, pertan- to, ritenuti da questa classe inammissibili. Procopio racconta che durante un ricevimento nel gineceo, un patrizio si avvicina per chiedere ad un cortigiano dell'imperatrice di onorare un debito contratto. Tuttavia la sovrana, anziché ascoltare la legittima pretesa del patrizio, preferisce umiliarlo senza riguardo per la sua posizione. Teodora, a dire di Procopio, così sembra esordire:

\footnotetext{
"Patrizio tal di tali" e il coro degli eunuchi proseguì sulla stessa aria: "hai una grossa ernia". Quello supplicò di nuovo e pronunciò un discorso analogo al precedente, ma la donna rispose allo stesso modo e il coro riprese le parole finché il poveretto, rinunziando, fece l'abituale reverenza e se ne tornò a casa (Proc. Caes., Hist. Arc. V, 25-35).
}

Un'offesa irrimediabile per la classe dominante, che avverte la messa in discussione delle prerogative del proprio status. La nobiltà attraverso la circolazione di un'opera a destinazione elitaria come la Historia Arcana porta in essere un sommesso attacco alla monarchia, colpendo il soggetto più vulnerabile che la rappresenta. Questa diventa anche il 'capro espiatorio' di una strategia di autorappresentazione che non teme di nascondere dei risvolti assolutistici. L'odio dell'aristocrazia alla luce di quest'ultimo fatto, sempre se vero, è forse minimamente giustificato. Anche se pare liquidabile piuttosto come un mero espediente volto a fomentare sentimenti avversi alla casa imperiale ed a coalizzare i membri della classe dominante attorno a tale causa.

\section{GLI ANEDDOTI IMBARAZZANTI DELLA VITA PRI- VATA DELLA FAMIGLIA IMPERIALE E LA KAISER- KRITIK. LA MALATTIA DEL SOVRANO, UN PROBLE- MA RETORICO?}

L'Historia Arcana con la sua serie di aneddoti imbarazzanti non può essere liquidata come mera espressione dell'attività critica di un'aristocrazia che ha subito l'ingerenza dell'imperatore, ma configura piuttosto la prova della sofferenza patita dalla stessa classe dominante a causa degli atteggiamenti assolutistici assunti da parte della monarchia (Wirth; Haury 1963, pp. 283-284). Tutte queste istanze si saldano in una puntuale critica avverso la politica dell'imperatore. A maggior ragione, allorché Giustiniano pretende di essere acclamato con il titolo di despotēs, mentre l'imperatrice vanta per sé il titolo di despoina (Proc. Caes., Hist. Arc. XXX, 21-26). Questa politica di autorappresentazione ha un ovvio corollario: ai magistrati, specularmente, corrisponde il titolo di "servi". Si raffronta una fin troppo palese limitazione delle fa- 
coltà in cui incorrono non solo le magistrature, quali organi dell'Impero, ma piuttosto l'aristocrazia intera che riveste tali cariche. Con l'assunzione della nuova titolatura viene a cadere l'ultimo limite formale che si oppone alle pretese di onnipotenza dell'istituzione monarchica, che perde definitivamente la maschera democratica con cui Augusto ha celato l'essenza stessa del principato. Giunge al suo compimento un processo che formalmente inizia con l'altezzosa scelta di Diocleziano, allorquando questi pretende per sé la proskýnesis, il fasto dei sandali di porpora e delle pietre preziose. Diocleziano pone in essere una "commedia" che è la più diretta espressione del 'teatro del potere' e si oppone a quell'altra "commedia", sebbene più "nobile", "virile" ed "affettata" perpetrata sotto Augusto. ${ }^{5}$

Una pretesa che a Procopio sembra assurda, allorché Giustiniano introduce il bacio del piede, quale privilegio concesso dall'imperatore all'aristocrazia:

Un tempo il Senato, quando si presentava al sovrano, era solito ossequiarlo in questo modo. Un patrizio si inchinava fino alla mammella destra dell'imperatore e questi lo baciava sul capo e lo congedava. Tutti gli altri piegavano il ginocchio destro davanti al sovrano e se ne andavano (...). Tutti quelli che erano ammessi alla presenza di Giustiniano e Teodora, tutti indistintamente e anche coloro che avevano la dignità di patrizio, si gettavano subito a terra bocconi e, tenendo mani e piedi, sfioravano con le labbra il piede di entrambi e quindi si alzavano (Proc. Caes., Hist. Arc. XXX, 21-26).

Alla luce dei dati raccolti si può ritenere che la Historia Arcana configura un prodotto per l'uso e il consumo dell'aristocrazia, che mira alla demolizione della figura dell'imperatore, allorché pone sotto continuo attacco sia lui, che la sua sposa. Ciò al solo scopo di delegittimare la sua azione, mettendo in luce una certa inadeguatezza del sovrano (Ravegnani 1989, p. 50; Ravegnani 2008, p. 135).

Eppure, molti di questi aneddoti non sembrano essere dotati di particolare pregio, ma possono essere liquidati come esagerazioni tendenziose e, pertanto, vanno ricondotti ad un disegno politico; non hanno perciò un semplice intento narrativo. Queste esagerazioni, dunque, sembrano limitarsi a restituire il punto di vista di un'aristocrazia scontenta, che ha ordinato a Procopio un'opera altamente polemica. A tramite di questo testo l'aristocrazia sommessamente si oppone alla monarchia e tenta di destabilizzarla.

Non c'è però da stupirsi che alcuni di questi aneddoti imbarazzanti possano essere mutuati dall'agio- grafia. Devono però essere reinterpretati ed hanno a perdere la loro carica polemica, per divenire, questa volta, strumenti ad uso e consumo della Chiesa.

Il locus malizioso, partendo dalla cattiva fama di Teodora, permette persino di sostenere la plausibilità dell'ipotesi di una malattia sessuale contratta dall'imperatore. Nonostante l'intento di simili scelte letterarie, si nota come in modo paradossale quegli aneddoti hanno ad affermare i valori della monarchia. Anzi, attraverso l'adynaton viene garantita l'adeguatezza del sovrano, perché il meraviglioso conferma il consenso divino in favore dell'imperatore; ne costituisce così la prova provata. Al contempo, quest'augusto soccorso dalla divinità cristiana viene posto in uno 'spazio di copertura', in cui l'opera del Dio svolge una funzione legittimante; la benevolenza celeste poi si oppone alle critiche dell'aristocrazia e le priva di pregevolezza fattuale.

Si comprende bene come la menzione di una malattia sessuale non possa trovare spazio nella biografia ufficiale del sovrano, nonostante l'utilità appena adombrata. Quale locus letterario può ottenere, a prescindere dalla specifica patologia, maggiori possibilità di successo nella letteratura agiografica, laddove conferma, con l'adeguatezza dell'imperatore nel suo ruolo, la potenza taumaturgica del santo.

Un testo agiografico del sec. VI, come la Vita di San Sansone ospitaliere, ci informa che "una grave malattia colpì Giustiniano agli organi genitali e si manifestarono ulcere incurabili alla vescica" (Vit. S. Sam., 283-284). Molti medici hanno visitato l'augusto, ma nessuno ha una cura adeguata per lui. Sempre stando al succitato testo, la malattia fortunosamente viene curata dall'intervento di San Sansone. ${ }^{6}$ Questo adynaton ovviamente è fondamentale nella narrazione della vita del monaco Sansone, perché dimostra il potere taumaturgico del santo, che si oppone ad una malattia sconosciuta ed incurabile, come quella che, a dire dell'agiografo, produce ulcere nella vescica dell'imperatore. Al contempo, l'adynaton si dimostra altrettanto indispensabile per la monarchia e mostra come il sovrano possa trovare giovamento pure da un fatto imbarazzante. Ciò in ragione del favore divino esplicato nei suoi confronti, che pone rimedio ad una situazione innanzi a cui le conoscenze mediche degli uomini sono impotenti.

Körbler, al contrario, interpreta la malattia sofferta da Giustiniano quale uretrite gonococcica o saprofitica, che è guarita spontaneamente, senza bisogno di alcuna cura particolare (Körbler 1974, pp. 15-22). Tuttavia, non è agevole far collimare la teoria di Körbler con i dati evincibili dalla fonte agiografica. Questa tesi forza eccessivamente il quadro clinico fornito dal testo. 
Prima di tutto, la malattia menzionata dalla Vita di San Sansone presuppone la presenza di ulcere alla vescica. L'assenza di strumenti endoscopici lascia pensare che le ulcere alla vescica siano state meramente supposte solo in base alla sintomatologia. Quindi l'agiografo ha ritenuto opportuno aggiungere questo dettaglio, partendo dalla semplice conoscenza di una sintomatologia afferita ad una variegata casistica di patologie da lui conosciute. Forse proprio questo dato può giustificare il fatto che si faccia riferimento ad una malattia altrimenti identificata, sicché lo stesso autore non si sbilancia nell'offrire un'anamnesi puntuale del caso. Anzi, il fatto che la malattia sia sconosciuta ai medici e, pertanto, incurabile aumenta il pathos narrativo e dà prova della potenza del santo contro le miserie degli uomini. Un espediente dunque, che si risolve entro il piano narrativo. Perciò, in mancanza di un più preciso quadro clinico, siamo nel campo delle semplici presunzioni. Tanto basta all'agiografo per suggestionare il proprio pubblico.

Eppure, l'afferirsi alla presenza di ulcere nella vescica trova riscontro nella più tarda trattatistica di un noto medico bizantino come Leone, operante nel IX secolo d.C.. Costui afferma che le ulcere appaiono nella vescica allorché i pazienti presentano la disuria, ovvero quando effettuano la minzione in modo doloroso. ${ }^{7} \mathrm{La}$ biografia di San Sansone perciò precisa la presenza di ulcere, quale causa più probabile del malessere che affligge l'imperatore. Nondimeno, data la sintomatologia fin troppo generica, non si comprende come Körbler possa ricondurre la patologia ad un'uretrite gonococcica o saprofitica (Körbler 1974, pp. 15-22).

Il locus agiografico della malattia sconosciuta che colpisce l'imperatore ed i suoi sintomi, tali da coinvolgere la zona genitale, non possono non avere implicazioni rispetto al contesto a cui l'opera si rivolge. Difatti è noto che tale letteratura risponde ad un'esigenza edificante e propone all'immaginario collettivo la visione di un universo dominato dal Logos divino, che razionalizza ogni possibile accadimento entro un preciso ordine, nonché contempla per le umane azioni delle giuste retribuzioni divine. Qui nulla è lasciato al caso, ma su tutto domina il Dio cristiano, il quale permette a qualsivoglia soggetto di sperimentare il male al solo scopo di poter espiare i peccati commessi, sia quelli noti a tutti, sia quelli conosciuti solo dalla divinità. II locus della Vita di S. Sansone che concerne una possibile patologia di Giustiniano investe allora un problema fondamentale per la cronologia di riferimento: il rapporto fra l'uomo e l'ordine divino, a maggior ragione se racconta la dialettica delle interazioni fra il 'sovrano del cielo' e quello della terra. La prefata Vita offre in via incidentale ed a latere alla narrazione delle gesta del santo, un affresco che contempla alcuni precetti fondamentali delle elaborazioni cristiane, come il libero arbitrio, da contemperare con le nozioni di giustizia e misericordia divina (Amundsen 1996; Marinis 2016; Lymberopoulou 2020). Precetti che si oppongono ad una percezione diffusa del mondo e condivisa anche dal quisque de populo, giacché si muove in parallelo ai prodotti della cultura cristiana e si richiama alle categorie cognitive gentili. Si evoca in particolare l'idea di un fato che incombe e schiaccia l'uomo, lasciandogli davvero poco spazio per agire. E se la tradizione cristiana in via di consolidamento propone il suo kaıpós tutto compenetrato dalla divinità, deve altresì contrapporsi a tale visione la nozione del xpóvos in cui domina l'uomo, il quale tenta di razionalizzare il proprio rapporto col divino entro la dimensione concessa alla sua azione. Si raffronta uno spazio che si colora di tinte fosche e funge da scenario ad una serie di temi ampiamente sviluppati dal genere della tragedia. Sicché si può dire che la tragedia classica con il suo iter di espiazione dell'errore spesso vissuto in solitaria si contrappone ai processi di ká $\theta \alpha \rho \sigma \iota \varsigma$ cristiani, che vengono sempre catalizzati dall'azione dell'uomo di Dio, il quale intercede in favore del singolo o della comunità.

E se il pensiero cristiano concepisce una raffinata casistica di peccati che hanno a coinvolgere sia il foro interno, sia quello esterno, di cui si deve sempre dare conto a Dio, i precetti della religione gentile metabolizzati attraverso lo strumento aulico di comunicazione della tragedia e proposti all'audience, offrono una visione ben diversa. Si raffronta nel contesto della tragedia classica la centralità dell'uomo e della sua azione, che può essere anche disinvolta. Eppure essa si infrange sull'insondabile limite costituito dal volere degli dei e da una serie altrettanto rigida di tabù rituali. Tant'è che questi può incorrere nell'errore, che potremmo definire hamartia, allorché lo si declina in termini aristotelici (Arist., Poet. 1453a, 5-20). ${ }^{8}$ A suo corollario l'uomo comune, allorché condivide tali valori, ha ad avvertire una sorta di lacerazione interna, dovuta alla percezione della limitatezza che lo inficia. La tensione prodotta dalla texture di sensazioni viene poi sublimata entro l'armoniosa struttura del racconto tragico. Tale genere teatrale, stigmatizzando il senso del limite antropico, la cui infrazione può addirittura essere inconsapevole, sanziona la condotta dei personaggi anche a prescindere dalla volontà del soggetto agente e dalla consapevole violazione operata da costui. Le conseguenze dell'errore, sublimate dalla visione edulcorata di quel genere teatrale, lasciano sperimentare all'audience, nell'ambito dell'azione scenica e durante il tentativo posto in essere dai personaggi e volto a 
superare l'umano limite, le possibili soluzioni a questa dicotomia esistenziale.

Pertanto è possibile paragonare la disinvolta condotta di Giustiniano, che viola consapevolmente i valori della classe a cui appartiene, sposando una donna di rango inferiore, e quelli della gerarchia cattolica, allorché offre il suo incondizionato appoggio alla moglie di fede nestoriana, all'azione meno consapevole, ma altrettanto contraria ai valori condivisi e diffusi dalla cultura greca, posta in essere dall'Edipo re.

Gli eccessi nelle passioni portano alla violazione dei valori condivisi dal sistema di riferimento e costituiscono il presupposto del $\mu i \alpha \sigma \mu \alpha$ che investe l'agente. Tant'è che attirano la punizione divina, la quale si concretizza nella malattia atta a colpire l'area del corpo adoperata per soddisfare tali pulsioni. La mediazione

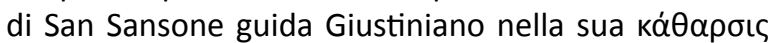
e culmina nella guarigione ottenuta a medio della sua intercessione. Nella tragedia classica il personaggio di Edipo invece si dimena tra la resistenza al destino, presagito dall'oracolo che predice l'omicidio paterno e l'incesto, quale squarcio visivo orribile sul futuro prospettato all'audience, e l'ineluttabile compiersi di tali eventi. ${ }^{9}$ Sicché il protagonista si pone di fronte all'inevitabile infrangersi di un tabù culturale, che attira su di lui il biasimo comune. Tuttavia, tale trasgressione è solo parte del tragico destino di quello che dal punto di vista morale è qualificato come uomo "medio" (Arist., Poet. 1452b, 31-1453a, 23). Costui è piuttosto l'"eroe dell'intelligenza", che conquista il potere su Tebe svelando l'enigma della Sfinge. II consumarsi della trasgressione sessuale, difatti, richiede un prezzo da pagare, mentre la sua colpa si pone all'origine del $\mu i \alpha \sigma \mu \alpha$ che "contamina" Tebe (Sof., Edip. vv. 130-138; Bettini; Guidorizzi 2004). Eppure, anche di fronte a tale evento Edipo non rinuncia ad esercitare il suo intelletto, vuole fare luce e guardare oltre il velo dei fenomeni per comprenderne le cause più intime. Il superamento delle convenzioni stabilite dalla morale condivisa, unito all'esercizio della coscienza critica, che tenta di superare il limite di comprendonio concesso agli uomini è causa della rovina del protagonista e funge da motore dell'azione scenica (Lombardi 2016, pp. 102-104; Segal 1981; Segal 1995; Winnington-Ingram 1980). II testo tragico propone allora un'ambigua ironia: il progredire nella conoscenza delle cause del $\mu i \alpha \sigma \mu \alpha$ cittadino corrisponde ad un'introspezione nel sé ed ad una presa di coscienza della condotta nefasta da parte di Edipo. Tuttavia, quella tragedia non contempla il superamento della colpa attraverso la kó $\theta \alpha \rho \sigma \iota s$, come invece accade nel racconto agiografico. Nonostante la cultura greca conosca i Riti eleusini, gli eventi tragici si consumano con la perdita della vista del protagonista, quale rituale di espiazione e quale sorta di contrappasso ante litteram. Ciò consta nella privazione dell'organo sensorio con cui si è visto quel che è tabù (Sof., Edip. vv. 981-83; 1334s). Ma non solo. La di lui vista viene colpita anche perché costui, attraverso l'oracolo, ha osato portare il proprio sguardo in avanti -ed indi il suo comprendoniooltre il velo opposto dalla fenomenologia.

In entrambi i casi presi in considerazione si nota l'intimo legame tra la pulsione sessuale e la messa in discussione dell'ordine familiare e dei valori del lignaggio, la cui sovversione porta disordine. E se la lascivia di Giustiniano orienta i suoi appetiti verso una donna non degna del rango imperiale ed è la probabile causa di una qualsivoglia malattia sessuale, le umili origini di quella stessa donna e la sua disinvolta condotta nella gestione degli affari di governo sono causa di contrasti con la classe nobiliare. Al contrario, la sovversione dei tabù sessuali ed il tentativo di aggirare il limite antropico in ultima analisi scatenano in Tebe la pestilenza. II racconto agiografico e la tragedia narrano, muovendosi entro le formule proprie del genere letterario a cui appartengono, un iter conoscitivo percorso da colui che detiene il potere temporale, il quale sperimenta attraverso il dolore, sia esso fisico o psicologico, la dicotomia tra essere e dover essere, tra apparire ed identità. Sicché l'io dissestato dalle pulsioni dei protagonisti si riflette su un mondo che soffre le conseguenze delle azioni avventate degli uomini. Entrambi i racconti hanno una funzione antropologica e psicanalitica e devono coinvolgere l'audience mettendo a nudo le loro passioni, allorché propongono il tentativo di superare quel "disordine" nei rapporti familiari che i due sovrani non riescono "a riassestare" (Parker 2011, pp. 11-26). E se la dicotomia $\mu i \alpha \sigma \mu \alpha / \kappa \alpha ́ \theta \alpha \rho \sigma \iota \varsigma$ viene risolta in favore dell'imperatore cristiano dall'intervento del santo, una felice conclusione di un tale processo dialettico non viene concessa all'uomo qualsiasi e nemmeno ad Edipo, sebbene egli detenga il potere. Manca nell'ultimo caso citato il momento di sintesi agevolato dall'alto, mentre il personaggio tragico si scontra con il limite antropico costituito dall'ineluttabilità del destino. $E$ se gli eccessi delle passioni sono quasi stimolati dalla detenzione del sommo potere, ad essi si deve necessariamente opporre un potente contrappeso consistente nel $\mu i \alpha \sigma \mu \alpha$, che investe il soggetto agente e stigmatizza la di lui condotta. Cosa che non è senza conseguenze, ma ammorba tutti gli aspetti della loro vita, fin tanto da investire i rapporti di forza, che sono alla base del potere detenuto da quei governanti. Ne consegue la radicale messa in discussione delle relazioni di soggezione, la necessità di rinegoziare tali situazioni e di rifondare su presupposti tutti nuovi l'insieme di 
relazioni di cui è protagonista il sovrano a mezzo dello

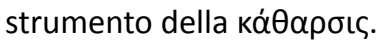

Al contempo, deve poi considerarsi l'ipotesi subdolamente proposta da Procopio nella sua Historia Arcana e concernente una malattia non specifica, che uccide l'imperatrice. Questi ha pure cura di descrivere il viso pallido della donna durante gli ultimi anni di vita e puntualizza che Teodora con l'età vede venire meno la sua bellezza. Lo confermerebbero pure i suoi ritratti, come il famoso mosaico nella Chiesa di San Vitale a Ravenna, laddove l'imperatrice è raffigurata malinconica, con un'espressione patetica e di stanchezza (Galavaris 1978, pp. 390-391). Mirando il mosaico ravvenate colpisce il "volto maestoso e triste, per non dire funereo, dell'imperatrice", che si oppone alla serena ieraticità dei compagni e delle compagne; ciò ha fatto pensare ad un'effige post-mortem della sovrana. ${ }^{10}$ Procopio insinua pure che la malattia capace di portare alla morte Teodora, non ha affatto migliorato l'irascibilità del suo carattere. Nondimeno, appare davvero difficile affermare che il decesso dell'imperatrice sia conseguenza di una qualsivoglia malattia sessuale. Anzi, si mostra del tutto irragionevole in assenza di un più completo quadro clinico.

Nonostante la vaghezza dei dati diagnostici, la teoria di Körbler attribuisce la morte di Teodora a un attacco di rupia sifilitica, che ha colpito le sue ghiandole mammarie; patologia accompagnata da un'eruzione di pustole (Körbler 1974, pp. 15-22). Un'ipotesi che non tiene conto degli ulteriori dati evinti dalle fonti.

Un vescovo africano, Vittore Tonnennense, contemporaneo dell'imperatrice afferma nella sua Cronaca che Teodora è morta a causa di un cancro al seno. La malattia ha prodotto metastasi diffuse per tutto il suo corpo: "Theodora Augusta Calchedonensis Synodi inimica canceris plaga corpore toto perfusa vitam prodigiose finivit". ${ }^{11}$ Una tesi, quella del cancro al seno, che viene ritenuta piuttosto plausibile dalla dottrina.

I medici romano orientali conoscono bene questo tipo di patologia e la sua sintomatologia. Pertanto, è difficile che un uomo erudito come Vittore Tonnennense li confondesse. Ezio di Bisanzio, un medico del sesto secolo, e Paolo di Egina, un medico del settimo secolo, hanno entrambi conoscenza della sintomatologia del cancro al seno e lo considerano la malattia più comune che affligge la donna, insieme al cancro dell'utero. ${ }^{12}$ Descrivono anche tecniche per la rimozione dello stesso, proponendo interventi chirurgici che possono prevedere sia l'asportazione totale, sia quella parziale della mammella malata. Ezio, in particolare, offre uno spaccato delle conoscenze del tempo e dimostra come la speculazione teorica concernente questa specifica patologia sia piuttosto raffinata. Questi poi avverte che il carcinoma mammario è inoperabile allorché aderisce al torace, tanto che la sua rimozione configura un'operazione alquanto pericolosa e quindi sconsigliabile..$^{13}$ Diversamente, un eminente medico del quarto secolo, Oribasio, raccomanda solo trattamenti atti alla conservazione. $^{14}$

L'approfondita conoscenza della patologia lascia presupporre che i medici del Palazzo siano in grado di identificarla molto facilmente, tanto da poter offrire trattamenti, anche chirurgici, adeguati al caso. La mancanza di ulteriori indizi nelle fonti storiche permette di ipotizzare che il cancro di Teodora sia del tipo inoperabile. Queste informazioni da sole tolgono ogni pregevolezza alla tesi che vuole la morte dell'imperatrice causata dalla sifilide (Diehl 1908, pp. 51-52; Nicol 1993, p. 124).

Alla luce di quanto dinnanzi diventa davvero difficile credere all'ipotesi di Körbler. È altrettanto difficile sostenere che Giustiniano abbia contratto la sifilide in una cronologia così precoce come la fine della Tarda Antichità o la Proto Bisanzio (Lascaratos; Poulakou-Rebelakou 1999, pp. 789-791). Deve poi aggiungersi un ulteriore dato che toglie verosimiglianza all'ipotesi. Niceforo Callisto Xantopulo, un ecclesiastico del XIV secolo, che è in possesso di accurate informazioni sui fatti riguardanti Giustiniano, tratte da fonti ben più antiche, afferma che la malattia dell'imperatore viene identificata con "una litiasi della vescica causata dalla sua dieta poco sana e dalla fatica". ${ }^{15}$ Questa affermazione da sola sembra negare qualsiasi valore all'ipotesi che sostiene la contrazione di una qualsivoglia malattia sessuale. La patologia sembra causare "disuria, difficoltà nell'urinare e dolore, e il medico dell'imperatore non poté curare i suoi dolori insopportabili". ${ }^{16}$ Tale dato permette di postulare che il dolore sia causato da una colica, anche perché non sappiamo neppure se il dolore alla minzione fosse accompagnato dall'emissione di pus. Proprio la mancanza di pus va ad escludere la presenza di un batterio in particolare. Orbene, simili lacune di informazioni sconfessano l'infezione alle vie urinarie e sembrano invece corroborare l'ipotesi di una colica, rendendola ben più credibile.

Negli ultimi anni del suo regno, Giustiniano ha sofferto di una malattia alla gamba; patologia che sembra accompagnata dal solito dolore durante la minzione. Körbler interpreta questi sintomi e crede nella presenza di probabili edemi o noduli presenti sulle gambe dell'imperatore, additandoli a conseguenze della sifilide. Postula poi che quell'augusto abbia sofferto di pro- 
blemi di disuria (Körbler 1974, pp. 15-22). Entrambe queste ipotesi hanno suscitato la curiosità della letteratura scientifica e sono oggetto di dibattito. Procopio, che non dimostra alcun riguardo verso la sovrana, attesta però che la patologia alla gamba di Giustiniano viene curata con l'intercessione dei Santi Cosma e Damiano ed attraverso la pratica dell'incubatio. ${ }^{17}$ Prassi medico sanitaria antica, che prevede il riposo notturno in chiesa presso l'immagine del santo; si raffronta una consuetudine comune pure agli ebrei e praticata nelle sinagoghe. Procedura che si eredita dal culto di Asclepio e si completa con una visione onirica in cui il santo o la divinità interviene a curare o suggerisce essa stessa la cura (Edelstein et aliis 1945).

Ancora una volta l'ipotesi di Körbler sembra porre in essere una sovrainterpretazione dei dati in suo possesso. Questo perché la malattia alla gamba di Giustiniano non viene correlata da alcuno degli storici bizantini con la presenza di edemi o noduli. Quindi l'ipotesi di Körbler, che postula l'esistenza di gomme sifilitiche, deve essere necessariamente considerata una semplice congettura, mancando ogni dato a conferma del sintomo. Si deve poi tenere conto di un ulteriore indicatore: la fin troppo tardiva apparizione delle gomme sifilitiche, tipiche della sifilide terziaria. Queste di solito compaiono tra il terzo e il quinto anno dall'infezione e non certo dopo più di 28 anni dalla contrazione (James et aliis 1990, pp. 405-422). Bastano queste poche motivazioni per privare di ogni pregevolezza l'ipotesi di Körbler, che pertanto non è ritenuta affatto credibile. Un ulteriore dato utile a sconfessare tale tesi è fornito da Procopio, il quale afferma che l'imperatore ha sofferto di ripetuti e perduranti dolori al ginocchio. Possiamo così supporre che in età avanzata Giustiniano sia stato colpito da dolore artritico. Procopio inoltre informa il suo pubblico circa la dieta rigorosa riservata all'imperatore. Questa consiste semplicemente in acqua ed erbe selvatiche ed esclude la presenza del vino. Una dieta solitamente raccomandata dai medici bizantini ai pazienti che soffrono di gotta. Questa malattia è molto diffusa nella classe dominante di Bisanzio e quindi è ben nota ai medici, che ne descrivono dettagliatamente i sintomi nei propri trattati. Le misure prese dai medici evocano piuttosto un attacco di gotta e non fanno affatto pensare ad un'infezione batterica, anche generica. Le due malattie: gotta e litiasi del tratto urinario, sembrano poi essere molto comuni nella cronologia di riferimento, tanto che coesistono tra il $25 \%$ e il $50 \%$ dei casi, stando a quanto dedotto dalle diverse statistiche concernenti l'incidenza delle malattie (Lascaratos 1995, pp. 951-957).

Al contrario, la manifestazione di disuria può essere attribuita ad una formazione di calcoli nel tratto urina- rio (Lascaratos et alias 2001, pp. 631-634). I disordini urinari sembra che vengano curati dall'acqua del Monastero di Zoodochus Pege. ${ }^{18}$ II prefato Niceforo afferma che Giustiniano ha avuto una visione onirica della Theotókos. Costei in sogno gli consiglia di bere grandi quantità di acqua della sorgente del suo Monastero. Sembra altresì che la terapia abbia davvero funzionato, probabilmente perché ha causato la dissoluzione dei calcoli renali, poi espulsi con l'urina; tant'è che l'imperatore non ha più sofferto di quel dolore. Questo testo offre elementi per poter sostenere, senza dubbio alcuno, che la condizione di Giustiniano possa essere identificata con una litiasi della vescica; una malattia trattata sistematicamente come l'idropisia. Altra prassi sanitaria ben conosciuta dai medici romano orientali e perciò inserita nel testo a riprova della credibilità dell'assunto.

Più segnatamente, una situazione analoga a quella che vede San Sansone esprimere la propria potenza taumaturgica si pone a presupposto dell'azione dei Santi Cosma e Damiano, i quali intervengono a sanare la gamba di Giustiniano. La Madre di Dio poi sembra risolvere con successo una nuova malattia che colpisce l'imperatore nella zona genitale consigliandoli la cura adeguata. Ancora una volta il $\mu$ i $\alpha \sigma \mu \alpha$ causato dalla disinvolta azione di governo e il concedersi agli eccessi della passione richiede un'azione di kó $\theta \alpha \rho \sigma \iota \varsigma$ impossibile agli sforzi degli uomini comuni, ma agevolmente realizzabile attraverso l'attività dei santi martiri e l'infallibile patrocinio della Vergine. Siamo di fronte ad una serie di vicende connesse al meraviglioso talmente notorie e capaci di incidere l'inconscio collettivo, che non possono essere tralasciate e passare in sordina anche entro il contesto di un racconto sostanzialmente malevolo come la Historia Arcana. Sicché Procopio deve arrendersi all'evidenza dei fatti ed ammettere, che qualsivoglia episodio di sovversione dei rapporti alla base dell'esercizio dell'Impero da parte di Giustiniano, sebbene dovuto ai suoi eccessi, deve essere necessariamente superato in ragione dell'intervento divino. Questo agevola e realizza con la ká $\theta \alpha \rho \sigma \iota \varsigma$ dell'agente la rinegoziazione delle relazioni, nonché il ripristino del più generale equilibrio tra le parti sociali e tra aristocrazia e monarchia in particolare.

Si precisa poi che Procopio nel descrivere gli ultimi anni di Giustiniano adopera il solito carattere polemico. Afferma che l'imperatore ormai anziano mostra un comportamento bizzarro: appare nella notte e si aggira nel Palazzo come un fantasma o come un demone insonne. Procopio osserva: "alcuni di coloro che lo assistevano fino a notte inoltrata e vivevano con lui a Palazzo, persone di mente lucida, credettero di vedere al suo po- 
sto un fantasma di demone a loro insolito" (Proc. Caes., Hist. Arc. XII, 20).

Una descrizione prosastica capace di offrire ulteriori spunti sul quadro clinico e le patologie che possono affliggere in età avanzata Giustiniano. Un comportamento atipico, che diventa il punto di partenza per una più incisiva critica. Si sfrutta così lo stato di salute del sovrano e l'ipotetica presenza di una malattia mentale, al solo fine di dimostrare che è oramai inadeguato a governare. Procopio sferra allora un attacco diretto nel tentativo di delegittimare Giustiniano:

Si dice che un monaco assai caro a Dio, convinto da coloro che con lui abitavano nel deserto, si sia recato a Bisanzio al fine di intercedere per le genti che vivevano vicino alla comunità, vittime di maltrattamenti e di insopportabili angherie. Non appena arrivò fu ammesso alla presenza dell'imperatore ma, mentre stava per entrare, si ritrasse all'improvviso come ebbe superato la soglia con un piede. L'eunuco che lo aveva introdotto e i presenti insistettero a lungo perché entrasse ma egli, senza rispondere, quasi paralizzato se ne tornò nella stanza in cui alloggiava. Quando chi lo seguiva gli chiese perché si era comportato così, si dice abbia risposto apertamente di aver visto a Palazzo, seduto in trono, il signore dei demoni.... ${ }^{19}$

Ebbene, la suggestione provata dal santo monaco in visita al Sacro Palazzo, che addirittura vede sul trono il "principe dei demoni" e non il "pio" imperatore, appare come il più subdolo dei trucchi da retore che si può porre in campo (Proc. Caes., Hist. Arc. XIII, 2). Questo è un altro locus della kaiserkritik, che investe direttamente la teologia del potere, negando l'origine divina dello stesso.

Un'affermazione che contraddice pertanto i postulati della teoria ufficiale del potere, proposti dal diacono Agapito, il quale ha sistematizzato in un pensiero coerente tutte le diverse elaborazioni sul tema. Questi, al contrario, fa del sovrano romano un'emanazione stessa del Dio cristiano: "come l'occhio è parte integrante del corpo, così l'imperatore è integrato armonicamente nel mondo, concesso da Dio per collaborare con lui nel compimento di ciò che è vantaggioso (...). E' immagine di pietà fatta da Dio, (...) immagine vivente del Dio (...). ${ }^{20}$ Uno specchio terso (...) che brilla dei raggi divini (...). ${ }^{21}$ In questo potrà imitare massimamente Dio nel giudicare che nulla è più prezioso della misericordia (...), ${ }^{22}$ se fugge ogni atteggiamento contrario alla umanità come ferino ed esercita l'amore verso gli uomini come simili a Dio; ${ }^{23}$ abito che non invecchia è il manto della beneficenza; veste incorruttibile l'amore verso i poveri". ${ }^{24}$
A tramite di questo espediente Procopio vuol rappresentare al suo pubblico la superiorità morale dell'aristocrazia. Ciò comporta necessariamente la demonizzazione della monarchia assoluta e la dequalificazione dei suoi attributi. Stanti simili presupposti, vi è una certa difficoltà a credere che questi aneddoti rappresentino la realtà. Pertanto, si dubita del fatto che Giustiniano possa essere afflitto da qualsivoglia patologia mentale.

Dal punto di vista clinico si può sostenere che la strana condotta dell'imperatore, assimilabile al sonnambulismo o ad un'irrequietezza notturna, abbia a suggerire i sintomi della demenza senile. In verità, una qualche incapacità mentale sofferta dall'augusto viene già postulata da Nicolaus Alemannus, il primo editore della Historia Arcana di Procopio nel 1623; ciò ha lasciato pure pensare ad un sintomo della neurosifilide. Di conseguenza Körbler, sulla scorta di Nicolaus Alemannus, arriva a sostenere che Giustiniano abbia presentato sintomi di demenza senile durante i suoi ultimi anni (Körbler 1974, pp. 15-22).

Eppure, alcuno dei sintomi di senilità, che secondo la critica hanno afflitto Giustiniano, viene attestato dagli storici suoi contemporanei. Mancano inoltre informazioni particolareggiate nelle fonti circa lo stato di salute dell'imperatore nei suoi ultimi anni (Poulakou-Rebelakou et alias 2011b, pp. 405-412). Anche quella della demenza senile può essere semplicisticamente liquidata come una congettura. Poiché parte da un dato agevolmente riconducibile ad un mero locus polemico, inserito in un contesto per di più malevolo ed avverso ad una monarchia dai tratti assolutistici. Si raffronta un espediente di mera kaiserkritik volto a delegittimare la condotta piuttosto austera posta in essere dall'imperatore negli ultimi anni di vita. Sia i presunti sintomi di senilità, che l'incapacità mentale sono entrambi ulteriori loci utilizzati per destabilizzare la monarchia. Questi si aggiungono all'inadeguatezza morale di Giustiniano, che arriva a sposare una donna non degna del rango imperiale, ed all'impossibilità fisica e psichica al reggere da un certo punto in poi il governo dell'Impero. Un costrutto artificioso, ma non senza base fattuale, giacché sembra che l'imperatore sia stato afflitto da debolezza generale e soprattutto da mal di testa dopo il 560 d.C..

Gli storici a lui coevi sostengono che Giustiniano muore improvvisamente, senza alcun segno di particolare malattia; forse addirittura per cause naturali. Se accettiamo l'ipotesi di Körbler e crediamo in un vecchio Giustiniano che presenta sintomi di senilità alla fine della sua vita, rischiamo di andare incontro ad un'interpretazione eccessiva, che forza i dati del quadro clinico evincibile circa la salute dell'imperatore, divenuto ormai 
anziano. I sintomi presentati da Giustiniano e menzionati dalle fonti poi non sono sufficienti a dimostrare la contrazione della neurosifilide, in particolare di quella parenchimatosa e nemmeno della cosiddetta paresi. Mancano poi tutti gli altri sintomi di questa malattia come le idee grandiose, la megalomania e le allucinazioni, tutti tipici della neurosifilide. ${ }^{25}$ Le fonti non restituiscono nemmeno ulteriori tipi di sintomi, che si possano connettere alle altre diverse tipologie di neurosifilide. Indi per cui, si raffronta una mera congettura, priva di ogni pregevolezza.

\section{CONCLUSIONE}

Le fonti collazionate non solo permettono di aprire uno spaccato sulla vita intima della coppia imperiale, ma anche offrono importanti informazioni riguardanti la conoscenza medica della Proto Bisanzio. Circa la teoria di Körbler, ovvero la possibile contrazione di specifiche malattie sessuali da parte di Giustiniano e persino della sifilide, poco si può dire, poiché si è costretti ad ammettere che trattasi di ipotesi poco convincente. I sintomi sofferti dall'imperatore difatti sono fin troppo generici per dimostrare un quadro clinico puntuale. II dolore alla minzione solitamente può essere agevolmente associato ad una generica infezione batterica. Tuttavia, è davvero difficile credere che Giustiniano abbia contratto

\section{NOTAS}

1 Oriel 2012; Brondy 1937, pp. 2-106; Sigerist 1961; Wortley 2004, pp. 91-107; Angeletti 1992, pp. 207-218; Grmek 1989, pp. 133-151.

2 La singolare scelta di Giustiniano incontra il veto di Eufemia, l'imperatrice sua zia, moglie di Giustino I. II veto però sembra venire meno con la di lei morte nel 523, lasciandogli campo libero.

3 Potter 2015; Ziche 2012-2013, pp. 311-323; Browning 1987, pp. 165-178; Mantellou 1990, pp. 330-339; Ostrogorsky 1986, pp. 69-78; Hunger 1992, pp. 81-82.

4 Le elaborazioni politiche del periodo arrivano a sostenere una metafora che collega le insegne imperiali come la porpora e la corona alle virtù richieste al buon sovrano. Tant'è che l'imperatore deve cingersi della "corona della temperanza, rivestito della porpora della giustizia (...) parato della porpora dell'amore per i poveri". Cfr. Agap. Diac., Expositio capitum admonitorum, PG 86, cap. 18; cap. 60; Carile 2000, pp. 127-149; Patlagean 1992; Cavarra 1990, pp. 28-40.

5 Gibbon 1967, p. 346; Teja 1993, p. 613.

6 Halkin, François (1977-1978), "Saint Samson le xenodoque de Constantinople (Vle siecle)", Rivista di Studi Bi- la sifilide entro i limiti di quella precisa cronologia. La tesi di Körbler appare dunque sfornita di prove cliniche adeguate per essere dimostrata e di conseguenza viene liquidata come una semplice congettura. Appare più ragionevole sostenere che questa tipologia di sintomi, capaci di evocare malattie agli organi genitali, vadano interpretati come mero luogo letterario, volto a delegittimarlo e destabilizzare la monarchia. Eppure, questo non esclude in assoluto la contrazione di qualche patologia.

Deve rilevarsi che la tesi di una colica, che ha colpito Giustiniano in età avanzata sembra ben più credibile, certamente unita alla gotta, altra malattia alquanto comune nelle classi dominanti in ragione dell'alimentazione molto ricca. Una patologia probabilmente sofferta dall'imperatore, che può permettersi il dispendio di parecchie risorse per la propria mensa; tesi avvallata anche dalla dieta parca ed a base di verdure a cui questi viene sottoposto da un certo punto in poi.

Nondimeno, il riferimento ad una generica malattia dell'imperatore costituisce pure un locus che ha particolare successo in agiografia. L'adynaton e l'intervento divino sono utili alla monarchia, perché confermano la bontà intrinseca dell'istituzione imperiale ed affermano, infine, l'adeguatezza del singolo sovrano. L'infermità, orbene, giova alla figura dell'imperatore e la sua risoluzione diventa ulteriore prova della legittimità della sua azione.

zantini e Neoellenici, 14-16, pp. 5-17; Miller 1990, pp. 101-135.

7 Ermerins, Franz Zacharias (1963), (ed.), "Leonis Philosophi conspectus medicinae". En: Anecdota Medica Graeca, Amsterdam, Hakkert, p. 193.

8 Gallavotti, Carlo (1974), (ed.), Aristotele, Poetica, Milano, Mondadori.

9 Avezzù, Guido; Guidorizzi, Giulio (2008), (eds.), Sofocle, Edipo a Colono, tr. it. G. Cerri, Milano, Fondazione Lorenzo Valla.

10 MacCormack 1995, p. 389. La nicchia su cui si staglia Teodora sembra rimandare alla sfera funebre. L'elemento architettonico viene adoperato per esaltare adeguatamente i trapassati, come dimostrano i documenti visuali siti nella stessa Ravenna, di cui si citano ex plurimis le nicchie con i profeti a Sant'Apollinare Nuovo e quelle con le effigi dei vescovi diocesani in Sant'Apollinare in Classe. Per le nicchie dei profeti in S. Apollinare Nuovo: Deichmann 1958, tavv. 100-107.

11 Mommsen, Theodor (1894), (ed.), "Victoris Tonnennensis episcopi Chronica". En: Monumenta Germaniae Historica, Vol. II, Berolini, p. 202.

12 Briau, Raoul (1855), Chirurgie de Paul d'Egine, Charlottesville, McLeod, pp. 210-213. 
13 Zervos, S. (1901), (ed.), Aetii Sermo Sextidecimus et Ultimus, Leipzig, Mangkos, pp. 60-68.

14 Raeder, Johannes (1933), (ed.), Oribasii Collectionum Medicarum Reliquiae. En: Corpus Medicorum Graecorum, vol. IV, Lipsiae-Berolini, Teubner, pp. 299-300.

15 Pamperis, A. (1802), Nicephori Callisti Xanthopouli about the Zoodochus Pege in Constantinople and its Miracles, Constantinople, pp. 14-15.

16 Ibidem; Poulakou-Rebelakou et alias 2011a, pp. 269-273.

17 Wirth; Haury 1963, pp. 56-68; 107-108; Cuscito 2007, pp. 99-111; Bucci 2016.

18 Pamperis, A. (1802), Nicephori Callisti Xanthopouli about the Zoodochus Pege in Constantinople and its Miracles, Constantinople, pp. 14-15.

19 Proc. Caes., Hist Arc. XXVIII; Ravegnani 1989, p. 41; Ravegnani 2016, cap. IV, nota 12 . II tema diventa un leitmotiv. Si giunge a sostenere che Teodora sogna di sposare

\section{BIBLIOGRAFÍA}

Amundsen, Darrel (1996), Medicine, Society, and Faith in the Ancient and Medieval Worlds, Baltimore, Johns Hopkins University Press.

Angeletti, Luciana R. (1992), "La medicina”. En: Settis, Salvatore (ed.), Civiltà dei Romani. Il rito e la vita privata, Milano, Electa, pp. 207-218.

Avezzù, Guido; Guidorizzi, Giulio (2008), (eds.), Sofocle, Edipo a Colono, tr. it. G. Cerri, Milano, Fondazione Lorenzo Valla.

Bettini, Maurizio; Guidorizzi, Giulio (2004), II mito di Edipo, Torino, Einaudi.

Briau, Raoul (1855), Chirurgie de Paul d'Egine, Charlottesville, McLeod.

Brondy, M.L. (1937), "The history of gonorrhea among the Greeks and Romans", Trans Am Neisser Medical Society, 3, pp. 2-106.

Browning, Robert (1987), Justinian and Theodora, London, Weidenfeld and Nicolson.

Bucci, Agapito (2016), I santi medici Cosma e Damiano, Roma, Armando Editore.

Carile, Rocco A. (2000), "Santi aristocratici e santi imperatori". En: Carile Rocco A. (ed.), Immagine e realtà nel mondo bizantino, Bologna, Scarabeo, pp. 127-149.

Cavarra, Berenice (1990), "Ideologia politica e cultura in Románia fra IV e il VI secolo", Quaderni della Rivista di Studi Bizantini e Slavi, Spoleto, CISAM, pp. 28-40.

Cuscito, Giuseppe (2007), “Origine e sviluppo del culto dei santi Cosma e Damiano. Testimonianze nella Venetia et Histria". En: Spadoni, Claudio; Kniffitz, Linda (eds.), San Michele in Africisco e l'età giustinianea a Ravenna, Cinisello Balsamo, Silvana, pp. 99-111.

Deichmann, Friedrich Wilhelm (1958), Frühchristliche Bauten und Mosaiken von Ravenna, Baden-Baden, Steiner. il principe dei demoni, cfr. Proc. Caes., Hist. Arc. XXXII; gli aneddoti di Procopio arrivano pure a suggerire che Giustiniano è un demone incarnato, cfr. Proc. Caes., Hist. Arc. XXVI; XXVIII; Rubin 1960.

20 Agap. Diac., Expositio capitum admonitorum, PG 86, cap. 5; Carile 2000, pp. 127-149; Patlagean 1992; Cavarra 1990, pp. 28-40.

21 Agap. Diac., Expositio capitum admonitorum, PG 86, cap. 9.

22 Agap. Diac., Expositio capitum admonitorum, PG 86, cap. 37.

23 Agap. Diac., Expositio capitum admonitorum, PG 86, cap. 40.

24 Agap. Diac., Expositio capitum admonitorum, PG 86, cap. 60.

25 Rook et allis 1986, pp. 839-859; James et aliis 1990 pp. 405-422.

Diehl, Charles (1908), Figures Byzantines, Paris, A. Colin.

Edelstein, Emma et aliis (1945), Asclepius: A Collection and Interpretation of the Testimonies, Baltimore, The Johns Hopkins Press.

Ermerins, Franz Zacharias (1963), (ed.), "Leonis Philosophi conspectus medicinae". En: Anecdota Medica Graeca, Amsterdam, Hakkert.

Galavaris, George (1978), “Early Byzantine art”. En: Christopoulos, George (ed.), History of Hellenic Nation, Vol. 7, Athens, Ekdotiki Athinon, pp. 390-391.

Gibbon, Edward (1967), Storia e decadenza dell'impero romano, Torino, Einaudi.

Gallavotti, Carlo (1974), (ed.), Aristotele, Poetica, Milano, Mondadori.

Grmek, Mirko (1989), Diseases in the Ancient Greek World, Baltimore-London, Johns Hopkins University Press.

Kaldellis, Anthony (2004), Procopius of Caesarea: Tyranny, History, and Philosophy at the End of Antiquity, Philadelpia, Penn.

Körbler, Juraj (1974), “Die Krebserkrankung der byzantinischen Kaiserin Theodora (Ein Beitrag zur Geschichte der Syphilis)", Janus, 61, pp. 15-22.

Halkin, François (1977-1978), "Saint Samson le xenodoque de Constantinople (Vle siecle)", Rivista di Studi Bizantini e Neoellenici, 14-16, pp. 5-17.

Hudson, Eugene (1965), "Treponematosis and man's social evolution", American Anthropologist, 67, pp. 885-901.

Hunger, Herbert (1992), Byzantine Literature, Vol. 2, Athens.

James, William et alias (1990), (eds.), Andrews' Diseases of the Skin. Clinical Dermatology, Philadelphia, Elsevier.

Lymberopoulou, Angeliky (2020), Hell in the Byzantine World: A History of Art and Religion, Cambridge, Cambridge University Press.

Lamma, Paolo (1947), “Giovanni di Cappadocia”, Aevum, 21, pp. 80-100. 
Lascaratos, John (1995), "Arthritis in Byzantium (AD 324-1453): unknown information from non-medical literary sources", Annals of the Rheumatic Diseases, 54, pp. 951-957.

Lascaratos, John; Poulakou-Rebelakou, Effie (1999), “Did Justinian the Great (527-565 CE) suffer from syphilis?", International Journal of Dermatology, 38, pp. 787-791.

Lascaratos, John et alias (2001), "Urolithiasis on the Byzantine throne", Urology, 58, 4, pp. 631-634.

Lombardi, Chiara (2016), "Shakespeare, Sofocle e gli archetipi del potere", Dionysus ex machina, 8, pp. 97-127.

MacCormack, Sabine G. (1995), Arte e cerimoniale nell'antichità, Torino, Einaudi.

Mantellou, P. (1990), "The personal life of Theodora before marriage with Justinian, according to Procopius Anecdota", Byzantine Studies, 2, pp. 330-339.

Marinis, Vaselios (2016), Death and the Afterlife in Byzantium: The Fate of the Soul in Theology, Liturgy, and Art, Cambridge, Cambridge University Press.

Miller, Timothy (1990), "The Sampson Hospital of Constantinople", Byzantinische Forschungen, 15, pp. 101-135.

Mommsen, Theodor, (1894), (ed.), "Victoris Tonnennensis episcopi Chronica". En: Monumenta Germaniae Historica, Vol. II, Berolini.

Nicol, Donald M. (1993), Biographical, Dictionary of the Byzantine Empire, Athens, Seaby.

Oriel, David J. (2012), The Scars of Venus: A History of Venereology, New York, Springer.

Ostrogorsky, Georgije (1986), History of the Byzantine State, London, Rutgers University Press.

Pamperis, A. (1802), Nicephori Callisti Xanthopouli about the Zoodochus Pege in Constantinople and its Miracles, Constantinople.

Parker, Jan (2011), "Introduction: Images of Tradition, Translation, Trauma". En: Parker, Jan; Mathews, Timothy (eds.), Tradition, Translation, Trauma: the Classic and the Modern, Oxford, Oxford University Press, pp. 11-26.

Patlagean, Évelyne (1992), Santità e potere a Bisanzio, Spoleto, CISAM.

Poulakou-Rebelakou, Effie et alias (2011a), "Urological diseases of the Byzantine emperors (330-1453)", Urology, 77, 2, pp. 269-273.
Poulakou-Rebelakou, Effie et alias (2011b), "Dementia on the Byzantine throne (ad 330-1453)", Geriatrics \& Gerontology International, 12, 3, pp. 405-412.

Potter, David (2015), Theodora: Actress, Empress, Saint, Oxford, Oxford University Press.

Raeder, Johannes (1933), (ed.), Oribasii Collectionum Medicarum Reliquiae. En: Corpus Medicorum Graecorum, vol. IV, LipsiaeBerolini, Teubner.

Ravegnani, Giorgio (1989), La corte di Giustiniano, Roma, Jouvence.

Ravegnani, Giorgio (2008), Imperatori di Bisanzio, Bologna, II Mulino.

Ravegnani, Giorgio (2016), Teodora, Napoli, Salerno editrice.

Rook, Arthur et aliis (1986), Textbook of Dermatology, Vol. 1, Oxford, Oxford University Press.

Rubin, Berthold (1960), Das Zeitalter Justinians, Berlin, de Gruyter.

Segal, Charles (1981), Tragedy and Civilization: An Interpretation of Sophocles, Cambridge, Mass.

Segal, Charles (1995), Sophocles' Tragic World: Divinity, Nature, Society, Cambridge, Mass.

Sigerist, Henry Ernest (1961), A History of Medicine: Early Greek, Hindu, and Persian Medicine, II, Oxford, Oxford University Press.

Wortley, John (2004), "Getting Sick and Getting Cured in Late Antiquity", Washington Academy of Sciences, 90-93, pp. 91-107.

Vertue, H.S.H. (1983), "Inquiry into venereal disease in Greece and Rome", Guy's Hospital Reports, 102, pp. 277-302.

Teja, Ramón (1993), "Il cerimoniale imperiale". En: Carandini, Andrea et alii (eds.), Storia di Roma, III, L'età tardoantica, Torino, Einaudi.

Winnington-Ingram, Reginald (1980), Sophocles: An Interpretation, New York-Melbourne, Cambridge University Press.

Wirth, G.; Haury, J. (1963), (eds.), “Procopii Caesarensis Anecdota (Historia Arcana)". En: Opera Omnia, Lipsiae, Teubner.

Zervos, S. (1901), (ed.), Aetii Sermo Sextidecimus et Ultimus, Leipzig, Mangkos.

Ziche, Hartmut G. (2012-2013), "Abusing Theodora: sexual and political discourse in Procopius", Buگ $\alpha v \tau \iota \alpha \kappa \alpha, 30$, pp. 311-323. 